\title{
Crystal Dehydration in Membrane Protein Crystallography
}

\author{
Juan Sanchez-Weatherby and Isabel Moraes
}

\begin{abstract}
Crystal dehydration has been successfully implemented to facilitate the structural solution of a number of soluble and membrane protein structures over the years. This chapter will present the currently available tools to undertake controlled crystal dehydration, focusing on some successful membrane protein cases. Also discussed here will be some practical considerations regarding membrane protein crystals and the relationship between different techniques in order to help researchers to select the most suitable technique for their projects.
\end{abstract}

\section{Keywords}

Crystal dehydration $\bullet \mathrm{HC} 1 \bullet$ Free mounting system $\bullet$ In situ $\bullet$ Membrane proteins $\bullet$ Relative humidity

\subsection{Introduction}

The effect of dehydration in crystal diffraction has been known since the beginnings of protein crystallography (Perutz 1946). It can often be detrimental to the crystal but also be very

The original version of this chapter has been revised. An erratum to this chapter can be found at DOI 10.1007/978-3-319-35072-1_13

J. Sanchez-Weatherby $(\bowtie)$

Diamond Light Source, Harwell Science and innovation Campus, Didcot OX11 0DE, UK

e-mail: juan.sanchez-weatherby@diamond.ac.uk

I. Moraes

Membrane Protein Laboratory, Diamond Light

Source/Imperial College London, Harwell Campus,

Didcot, Oxfordshire, UK

e-mail: isabel.de-moraes@diamond.ac.uk beneficial, dramatically improving diffraction properties. Partly exacerbated by the initial unavailability of cryo-cooling techniques, and tight control of water content in crystals prior or during data collection, dehydration has always been of paramount importance. In the early days, when most data were collected at room temperature, it was necessary to maintain the stability of samples for hours or even days (Pickford et al. 1993). This meant that researchers were more aware of the benefits and pitfalls of hydration control and accustomed to accounting for the role it played in their observed results. Dehydration has been reported to be beneficial on the diffraction quality of macromolecular crystals (Heras and Martin 2005; Newman 2006; Russo et al. 2012). In most cases the discovery has occurred as a result of casual observations (such cracked capillaries, 
drops left to dry, badly sealed trays and others) (Esnouf et al. 1998; Abergel 2004).

The changes caused by dehydration are of such importance that several past and present studies have focused solely in the understanding of alterations caused by dehydration and its implications on the functional interpretation of protein and DNA structures. (Biswal and Vijayan 2002; Kaushal et al. 2008; Saraswathi et al. 2002; Nagendra et al. 1998; Sukumar et al. 1999; Kuo et al. 2003; Bowler et al. 2006; Perutz 1946; Amunts et al. 2007; Dobrianov et al. 2001).

As X-ray sources became stronger, cryocooling rapidly became the norm (Hope 1988; Mitchell and Garman 1994; Garman 1999; Juers and Matthews 2004; Alcorn and Juers 2010). Once crystal samples are cooled, most of the alterations are prevented, so the need for careful control of humidity is diminished and shifted from the data collection stage to the pre-cooling sample preparation step (Farley et al. 2014; Pflugrath 2015). Crystal handling can have a significant effect on the hydration state of samples and, as it has been highlighted recently, it can have potential consequences in the structural solution (Farley et al. 2014). Therefore, crystallographers have a number of techniques and "tricks" in order to prevent these effects with greater or lesser success. Chemically, the cryo-protection process also causes alterations in osmotic pressure in the crystal samples, similar to a hydration/dehydration process, thus it can change the structure and packing, often leading to changes in diffraction quality (Heras and Martin 2005).

Despite documented benefits of controlled dehydration in macromolecular crystallography, dehydration is often not pursued due to a lack of well-established protocols and equipment. To address this, in the last few years a number of novel techniques have been made available, which focus on trying to make the experiments easier to carry out, reproducible and where possible, with X-ray feedback. These techniques range from simple tools to aid capillary mounting (Basavappa et al. 2003; Kalinin et al. 2005; Mac Sweeney and D'Arcy 2003; Yadav et al. 2005), to bespoke equipment designed to maintain a humid environment around the samples while mounted in the beam path of a synchrotron beam- line (Sanchez-Weatherby et al. 2009; Russi et al. 2011; Bowler et al. 2015; Kiefersauer et al. 2000a; Kiefersauer et al. 2000b; Kiefersauer et al. 2002; Kiefersauer et al. 2014).

The move to more complex crystallographic challenges and the availability of new techniques has meant more researchers are trying these experiments. As more experiments are being carried out and successes have been coming through, interest in crystal dehydration has been recently reignited (Moraes et al. 2014; Bowler et al. 2015; Kiefersauer et al. 2014).

This chapter will briefly review the current status of the field and the available tools, it will discuss the basic aspects of a dehydration experiment and give some guidance when attempting membrane protein crystal dehydration.

\subsection{The Dehydration Method}

As noted in the past (Newman 2006), dehydration can be a bit of a confusing term. Here we consider crystal dehydration as any process able to remove available water molecules from the crystals lattice. Dehydration is achieved by either altering the vapour (Sect. 6.2.1) or the chemical (Sect. 6.2.2) equilibrium of the crystal. These two methods of dehydration are different but also interrelated as the chemical alterations can be used to induce a change in the vapour environment and, the changes in the vapour surrounding the sample can induce alterations the chemistry. The relative amount of water present in a volume of air, Relative Humidity (RH), is directly linked to the temperature and chemical nature of a solution in equilibrium with the air. For this reason, salt solutions are universal calibrating standards for any equipment designed to measure air RH (Greenspan 1977).

When dehydrating crystals, it is advisable to try methods based on both approaches. In some cases, both methods can lead to very similar results but in others (particularly membrane protein crystals with detergents) the outcome of the process can be dependent on the particular technique used.

Crystallographers working in closed systems, like crystallisation plates or capillaries, can guide 
their dehydration experiments based on the wellestablished number of standard salts. Furthermore, the empirical equilibrium relative humidity when using the humidity control device (HC1) (Bowler et al. 2015; Russi et al. 2011; SanchezWeatherby et al. 2009) or the free mounting system (FMS) (Kiefersauer et al. 2002; Kiefersauer et al. 2000a, b) can be tabulated and tools exist that allow the researcher to try to estimate the theoretical alterations caused by chemical modification or the formulation of solutions required to induce a particular change in RH (Bowler et al. 2015; Wheeler et al. 2012).

\subsubsection{Dehydration by Modifying the Vapour Equilibrium}

This method is based on altering the water vapour pressure of the air surrounding the samples. This makes the water in the surrounding crystallisation buffer and crystal solvent channels equilibrate with the surrounding air making it saturate the air and lowering its availability in solution. Methods to carry this type of dehydration range from simple exposure of the samples to ambient dry air, through controlling the air environment using chemicals (Hellert et al. 2014; Kalinin et al. 2005; Kalinin and Thorne 2005), to the use of bespoke equipment designed to expose the samples to controlled dehydration (Bowler et al. 2015; Russi et al. 2011; Sanchez-Weatherby et al. 2009; Kiefersauer et al. 2002; Kiefersauer et al. 2000a, b).

\subsubsection{Dehydration by Modifying the Chemical Equilibrium}

Chemical alteration is achieved by the addition of a compound that will directly bond to the water molecules of the crystallisation buffer that surrounds the crystals. This lowers the number of interactions that proteins can establish with water, forcing it to interact with other molecules, thus inducing conformational changes. Chemicals used can include salts, precipitants and alcohols. From a practical point of view, the researcher either replaces the solution surrounding the crystals samples via careful pipetting or dialysis, or physically transfers the crystals into a new solution. This is the most frequent way of undertaking dehydration and it is often done in combination with cryo-protection or ligand binding. The key parameters in this process are the chemicals used, their concentration increment (if necessary) and the time samples are allowed to equilibrate in the new solution prior to harvesting (Shi et al. 2008; Adachi et al. 2009).

\subsubsection{Relative Humidity and Its Relationship with Cryo-Protection}

Key in dehydration experiments is the concept of Relative Humidity (RH). Relative Humidity is defined as the relative amount of water vapour in a given volume of air. It is expressed as a percentage of the saturation humidity and is pressure- and temperature-dependent (Winston and Bates 1960). As the chemical composition of a solution alters the saturation vapour pressure, it can be used to cross calibrate both the vapour and chemical dehydration experiments. For example at $20^{\circ} \mathrm{C}$ a saturated solution of $\mathrm{LiCl}$ is tabulated to be around $11 \% \mathrm{RH}, \mathrm{NaCl} 75 \% \mathrm{RH}, \mathrm{KCl}$ $86 \% \mathrm{RH}$, and $\mathrm{K}_{2} \mathrm{SO}_{4} 97 \% \mathrm{RH}$ (see Table 6.1

Table 6.1 Examples of equilibrium RH for a number of saturated salt solutions (Greenspan 1977)

\begin{tabular}{l|l}
\hline Saturated salt & $\mathrm{RH}$ at $20{ }^{\circ} \mathrm{C}$ \\
\hline Potassium hydroxide & 9.32 \\
\hline Lithium chloride & 11.31 \\
\hline Potassium acetate & 23.11 \\
\hline Magnesium chloride & 33.07 \\
\hline Sodium iodide & 39.65 \\
\hline Potassium carbonate & 43.16 \\
\hline Magnesium nitrate & 54.38 \\
\hline Sodium bromide & 59.14 \\
\hline Potassium iodide & 69.90 \\
\hline Sodium chloride & 75.47 \\
\hline Ammonium chloride & 79.23 \\
\hline Potassium bromide & 81.67 \\
\hline Ammonium sulfate & 81.34 \\
\hline Potassium chloride & 85.11 \\
\hline Potassium nitrate & 94.62 \\
\hline Potassium sulfate & 97.59 \\
\hline
\end{tabular}


Table 6.2 Examples of empirical equilibrium $\mathrm{RH}$ determined for the $\mathrm{HC} 1$ for a number non saturated salt solutions from (Bowler et al. 2015)

\begin{tabular}{l|l|l|l|l}
\hline Concentration $(\mathrm{M})$ & Sodium chloride & Sodium acetate & Sodium malonate & Ammonium sulfate \\
\hline 0.5 & 99.8 & 99.9 & 99.9 & 99.9 \\
\hline 1.0 & 98.9 & 98.9 & 99.3 & 99.1 \\
\hline 1.5 & 97.2 & 97.1 & 97.0 & 97.7 \\
\hline 2.0 & 95.3 & 94.8 & 95.2 & 95.9 \\
\hline 2.5 & 94.2 & 93.2 & 92.5 & 94.3 \\
\hline 3.0 & 92.8 & 91.2 & 89.9 & 92.8 \\
\hline
\end{tabular}

Table 6.3 Examples of empirical equilibrium RH determined for the HC1for a number standard precipitants from (Bowler et al. 2015)

\begin{tabular}{l|l|l|l|l|l|l|l|l}
\hline$\%(w / w)$ & PEG10000 & PEG6000 & PEG4000 & PEG1500 & PEG400 & PEG200 & Glycerol & Ethylene glycol \\
\hline 10 & 99.9 & 99.9 & 99.9 & 99.9 & 99.9 & 99.9 & 99.5 & 98.5 \\
\hline 20 & 99.9 & 99.9 & 99.9 & 99.9 & 99.9 & 99.5 & 96.5 & 94.0 \\
\hline 30 & 99.9 & 99.9 & 99.9 & 99.9 & 98.8 & 97.3 & 92.0 & 89.5 \\
\hline 40 & 99.3 & 99.9 & 99.2 & 98.3 & 96.3 & 94.9 & 88.5 & 85.0 \\
\hline
\end{tabular}

for a more comprehensive list). It is important to note that any standard calibration table applies to closed systems, therefore in devices like the $\mathrm{HC} 1$ and FMS that operate using an open airflow, the empirical equilibrium $\mathrm{RH}$ values are greater than those presented on Table 6.1 (see also Tables 6.2 and 6.3). For this reason the European Synchrotron Radiation Facility/the European Molecular Biology Laboratory - Grenoble Outstation (ESRF/EMBL) created a web tool (www.embl.fr/CrystalDehydrationCollaboration/ RH.html) that can be used to estimate the $\mathrm{RH}$ of solutions that includes theoretical and empirical values for better reference (Bowler et al. 2015; Wheeler et al. 2012). It is also important to remember that an individual crystallisation droplet will be in equilibrium with the well solution, but during the course of the crystallisation experiment the well might have dried up over time, thus it is much better to measure the empirical value of each tray before commencing an experiment.

In general, macromolecules tend to crystallise in highly hydrated solutions that require high $\mathrm{RH}$ to be stable but lack "cryo-protective" properties. Cryo solutions prevent ice formation by hydrogen bonding to the surrounding water molecules. These bonds also reduce the available water surrounding the crystal structure leading to a desiccation process. The empirical test of several precipitants commonly used in protein crystallisation clearly shows a correlation between the ability to cryo-protect and lower Relative Humidity (Wheeler et al. 2012). This highlights the intimate link between both effects (dehydration and cryo-protection) on crystal diffraction quality, however, it is difficult to understand the contribution from each of them when assessing diffraction quality collected at cryo-temperatures. Despite the key role dehydration may play in structure determination, researchers often do not carry out systematic studies on the effect it could have on the data quality, thus overlooking the role it might have played in their success or failure.

\subsubsection{Crystal Changes Induced by Dehydration}

The beneficial effects of dehydration are firstly, dependent on the original imperfections of crystal structure and then on the possibility of altering them by removing water molecules.

Macromolecular crystals are far from being "perfect", always presenting a certain degree of 


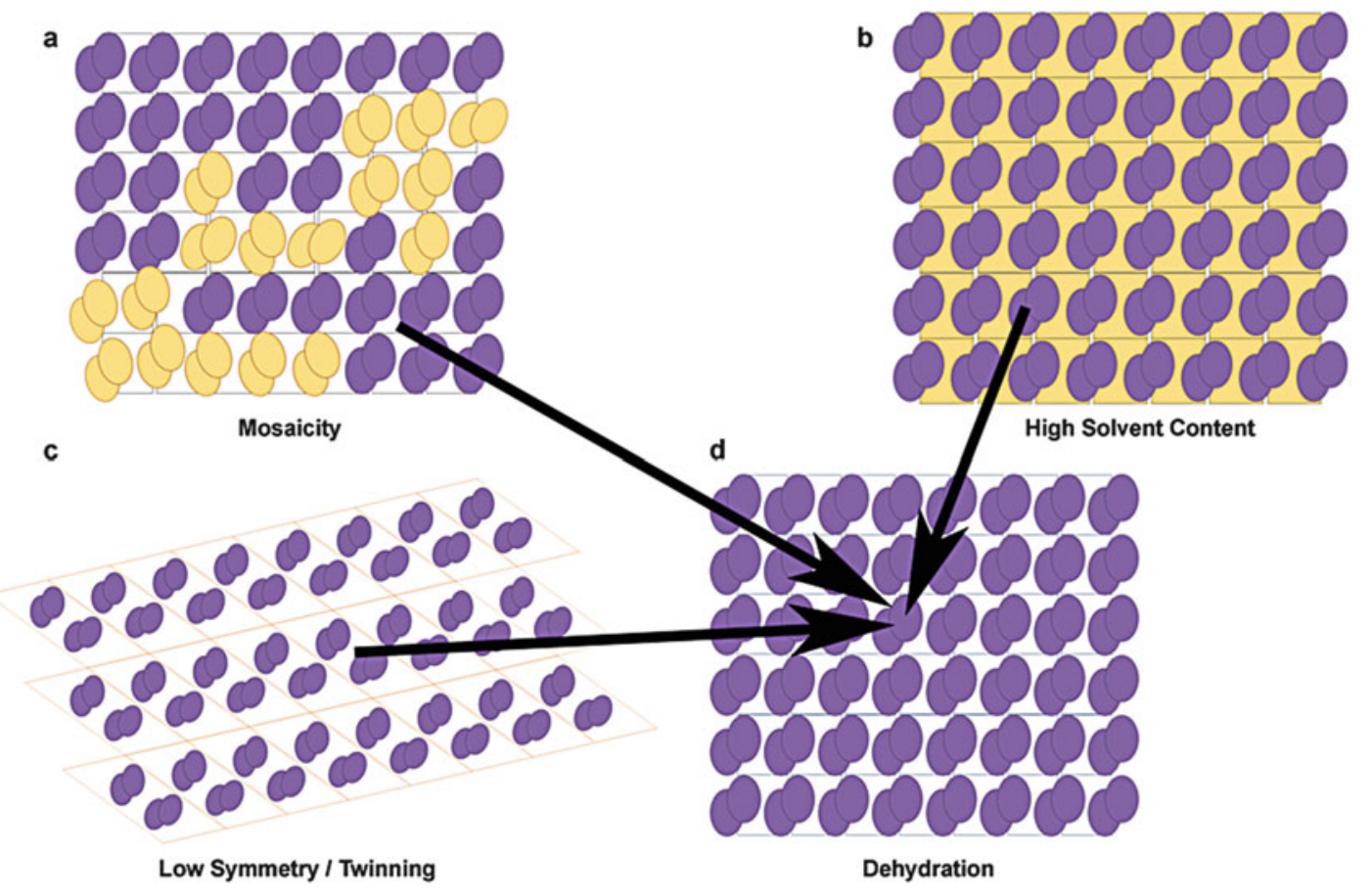

Fig. 6.1 Representation of possible lattice changes induced by dehydration. Most of these changes would lead to increased order or improved protein/solvent ration leading to improved diffraction properties. (a-d) Represents the reduction of crystal mosaicity.

mosaicity (meaning they lack of internal periodicity in certain areas called mosaic blocks), see Fig. 6.1. High mosaic spread in crystals it is mainly caused by the cryo-cooling process and a major contributor to the diffraction intensity reduction. Dehydration can reorganise these mosaic blocks, re-align them, and in principle leading to a crystal with better diffraction properties (Fig. 6.1a).

In other crystals, high solvent content also makes them scatter with less power. If by dehydration, one can lower their global solvent content maintaining the protein molecules order (Fig. 6.1b) (higher protein to solvent ratio) could lead to an improved diffracting power. Changes in solvent content need to be accompanied by small local rearrangements in the crystal contacts and/or flexible areas, therefore diffraction can improve as local motions are reduced and crystal structure changes. In these cases, the degree, direction and the energetics of these changes (b-d) Represents an increased or altered symmetry or the case of reduced twinning. (c-d) Shows the effect of reduced solvent bulk content leading to improved order or increased protein/solvent ratio

will condition whether changes are for the better. The actual physical change in the lattice may be subtle, but due to the diffraction phenomenon the results can be very dramatic.

The most dramatic cases are those where the alterations cause enormous difference in the lattice packing triggering a space group change. Often, as the molecules turn and twist, screw axes are generated or removed and alternated indexing solutions start emerging. These alterations might be positive if they induce greater symmetry.

Finally, as it was mentioned early in this section, poor diffraction often is associated with the physical alterations of the crystals when cooled to $100 \mathrm{~K}$. Nevertheless, the cryo-cooling process is very beneficial as it prevents radiation damage, lowers thermal motions of the molecules, reduces background and in certain cases also improves diffraction resolution. Dehydration prior to cryocooling in many cases helps to overcome the issues mentioned above as the pre-contraction 
induced by the dehydration process can yield a better lattice re-arrangement for the cryo-cooling. In other cases, dehydration is also beneficial as it can act as a way of cryo-protection (Pellegrini et al. 2011). Either by dry mounting, preventing ice forming around the crystals, or by concentrating the solutes within the solvent channels thus preventing hexagonal ice from nucleating in the solvent channels.

\subsubsection{Effect of Dehydration on the Membrane-Protein Crystal Lattice}

Membrane proteins can crystallise in two main forms depending on how they are grown. Crystals grown by the Lipid Cubic Phase (LCP) method (Caffrey 2003) tend to be formed by proteins organised in planar layers through proteindetergent-lipid hydrophobic interactions stacked on top of each other by polar interactions. These crystals are extremely small and fragile two dimensional plates known as type I 3D crystals. These crystals are difficult to harvest, difficult to see and most of their properties are intimately linked to the biochemical properties of the LCP. As the LCP structure is temperature and humidity dependent, this type of crystal is very unlikely to be useful for controlled dehydrations studies. On the other hand, membrane protein crystals can also grow using more "standard" crystallisation methods such vapour diffusion leading to crystals organised in a more three dimensional fashion (type II 3D crystals) similar to soluble proteins and more suited for dehydration experiments. Despite their similarity with soluble crystals, these membrane protein crystals tend to have lower protein content (10-25\% rather than 45$60 \%$ ) and higher solvent content (mainly water in soluble proteins) made up of detergents/lipids free micelles.

Integrity and dynamics of membrane proteins are closely related to the properties of the surrounding phospholipids in the native membrane. To avoid protein aggregation once removed from its natural environment, detergent is used in the protein solution in order to mimic the lipid membrane by surrounding the hydrophobic region of the protein generating a water-soluble protein-detergent complex (PDC). Therefore, membrane protein crystals formation and stability are strongly linked to the protein overall molecular structure (hydrophilic and hydrophobic exposed regions) and to the size, concentration and critical micelle concentration (CMC) of the detergent. While some membrane protein crystals are able to tolerate an increase or decrease of detergent once they have been formed others are more sensitive and lose their order if the equilibrium (bound detergent/free detergent) is changed. This will have a strong impact on the crystal dehydration ability. More information in the use of detergents in membrane protein structure determination can be found in Chap. 2 of this book.

In soluble protein crystals, dehydration tends to disturb the whole solvent volume and thus induce variation across the entire structure. Here, dehydration can affect solvent exposed areas, flexible loops, crystal contacts and internal hydrogen bonding networks. The idea behind dehydrating these crystals is to induce structural changes that will modify the packing (normally by altering crystal contacts or stabilisation of flexible areas) leading to better diffraction without negatively affecting areas that are already well ordered.

In membrane proteins, most of the interactions that keep the global lattice stable (via the detergent, lipid or interatomic interactions) are partially fluid and malleable. These regions are mainly hydrophobic and thus not severely affected by dehydration. The few hydrophilic interactions existent act as small anchors that stabilise the lattice. Humidity will affect these small soluble portions and induce changes that can propagate to the whole crystal via rearrangements on the global lattice structure, hopefully inducing a new ordered lattice that will promote better diffraction without affecting the integrity of the protein structure in study.

The magnitude of dehydration to induce a notable change on a crystal structure is, broadly speaking, different between membrane and soluble proteins. In soluble protein crystals, a small 
change in the global solvent content will cause a huge change in the whole crystal structure therefore these crystals usually require less dehydration. In membrane protein crystals, most of the alterations are targeted at the small solvent rich areas scattered around the lattice, and in order to remove enough water to successfully induce a change on the whole structure, the extent of dehydration needed is often much greater than for soluble proteins. The less amount of water in the lattice, the stronger the process needs to be to extract it. A good example of this are the crystals of the bile acid sodium symporter ASBT (Hu et al. 2011) that have required dehydration from an initial $\mathrm{RH}$ of $95 \%$ down to $45 \%$ in order to improve diffraction limit from around $8 \AA$ at room temperature to around $2 \AA$ after cryocooling.

Dehydration undertaken on membrane protein crystals without mother liquid in its surroundings (dry mounted samples - see Sect. 6.3.2) only induces changes in water content within the solvent channels. Note that although these channels have great amounts of detergent and other solutes there is very little water available for the dehydration process. On the contrary, dehydration methods that are performed on wet samples surrounded by their mother liquor (see Sect. 6.3.1) affect the whole drop. As water content is higher, dehydration will induce an increased concentration of solutes and detergent surrounding the crystals that can in turn alter the equilibrium with the crystal and disturb its order. For these reasons it is worth trying both wet-mounting and dry-mounting dehydration methods.

\subsection{Tools and Techniques for Crystal Dehydration}

Practical considerations and timing, determine what most researchers can do with their samples. Crystal availability and beam time do not always match and researchers will always struggle to get their best crystals ready in time for a dehydration study at a synchrotron or home source. In this section, the available dehydration methods and tools have been separated by the ability to execute them in a standard laboratory or at an X-ray source.

\subsubsection{Dehydration in the Laboratory}

Doing dehydration at the home laboratory has its advantages. The process can be performed using standard tools available within any molecular biology or biochemistry laboratory and with total control of the researcher. This also allows the researcher to test a great number of crystals (if available) without the usual time constrains during a synchrotron visit. In general, when dehydrating crystal samples in the home laboratory, the dehydration procedure is the most basic one where samples are just transferred from one solution to the other or exposed to air for a few seconds before proceeding to the cryo-protection process. This way of dehydrating is normally undertaken casually, not regarded important and often not well described in the methods sections of publications.

The most common dehydration techniques used in a standard laboratory are described below, see also Fig. 6.2.

\subsubsection{Simple Air Exposure}

This is the simplest dehydration method consisting in opening the crystallisation plate allowing the drops to dry for a certain amount of time prior to cryo-cooling (Fig. 6.2a). It is, of course, dependent on the ambient $\mathrm{RH}$, temperature, size of the drops and chemical composition of the crystallisation solution. It is mainly responsible for the "I am getting better at harvesting crystals" syndrome, as with time, crystallographers either start harvesting faster (allowing for less dehydration in delicate samples) or they harvest numerous samples from an individual drop (allowing for the last ones to dehydrate more). It can also be induced during the final harvesting prior cryocooling by allowing the sample to dehydrate on the loop before the final plunge in liquid nitrogen. Uncontrolled dehydration is difficult to avoid but careful note taking and repetition is the key to getting to control or understand its effects. As 


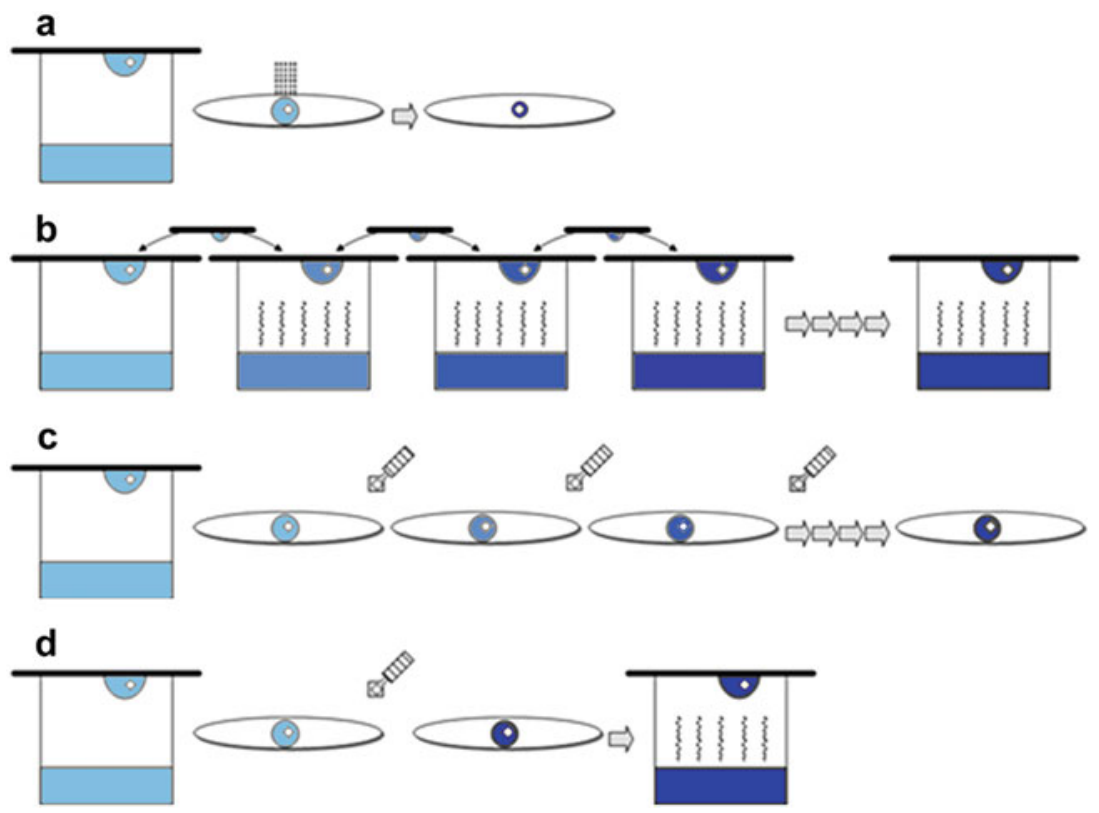

Fig. 6.2 Diagram showing alternate options for laboratory dehydration. (a) Simple air exposure, (b) vapour control by exchanging well solution, (c) chemical exchange

crystals become more fragile, smaller and grown in smaller volumes the effect of dehydration by air exposure becomes more prevalent.

\subsubsection{Vapour Control by Exchanging Well Solution}

This is probably the most controllable way of undertaking dehydration in the laboratory. It is, for obvious reasons, only possible in vapour diffusion crystallisation samples and relies on using the same crystallisation drop for the actual experiment. The idea is to replace the well solution with increased amounts of a desiccating solution (Fig. 6.2b). The new solution will lower the water vapour pressure and in return "pull" water out of the crystallisation drop inducing a change in the crystals. This is normally undertaken by using some of the standard saturated calibration salt solutions but can also be done using crystallisation precipitants. Depending on the volumes available, dehydration can be achieved by simple addition of salts to the pre-existing well buffer or by replacing the well with a totally new solution. Dehydration is a slow and gradual process most by serial soaking and (d) chemical exchange by soaking and slow equilibration

of the time, which depends on the drop size and well volume. It can be undertaken in one single step or in progressive steps and normally, after a period of equilibration (normally between 8 and $24 \mathrm{~h}$ ), samples are harvested and stored for data collection. The most attractive feature of this dehydration method is its simplicity and easy implementation. It can be undertaken whenever samples are available and once the optimal conditions are found it is very easy to reproduce. It can be achieved at any temperature and in general the presence of highly volatile compounds in the crystallisation solutions is not a concern.

\subsubsection{Dehydration by Chemical Exchange of Crystallisation Droplet}

As discussed earlier, this process is potentially happening during the standard cryo-cooling or soaking procedures (Fig. 6.2c,d). The main difference between this and the previous method described (Sect. 6.3.1.2) is that here it is possible to change the chemical components of the crystallisation drop and not only the concentration of 
the pre-existing ones. This procedure opens the possibility that the chemical used for dehydration alters the structure and/or the resulting diffraction by chemical modification rather than dehydration. In addition, the rate of exchange is more difficult to control and normally carried out by slow diffusion of buffers with increased concentration. If crystallisation droplets are very small, it is easy to cause a shock on the crystals, therefore premixing solutions to the target concentration can sometimes help. The simplest way of chemical dehydrating is by dialysis. This only requires replacing the solutions and, due to the dialysis membrane, it is very gentle and controllable.

\subsubsection{Modern Dehydration Methods with X-ray Feedback}

\subsubsection{Capillary Dehydration}

Dehydration performed in home laboratories, despite being the simple and cost effective, it is a partially blind process by the fact that is decoupled from the X-ray data collection. In addition, the cryo-cooling process undertaken after the dehydration procedure can muddle results.

This was noted early on in the use of macromolecular crystallography and initial attempts were made by developing tools that would couple salt-based vapour dehydration to the, then available, data collection using the capillary (glass or quartz) mounting method (Huxley and Kendrew 1953; Pickford et al.1993; Kiefersauer et al. 2000a, b). For a time, this proved to be useful (Rockland 1960; Dobrianov et al. 2001) but fell into disuse due to the high $\mathrm{X}$-ray scatter background produced compared to loops. The technique was re-introduced a few years later (Mac Sweeney and D'Arcy 2003; Basavappa et al. 2003) by adapting new lower background capillaries (made of a transparent polymer) to the then standard pinbased mounts (Kalinin and Thorne 2005; Kalinin et al. 2005; Hellert et al. 2014) (Fig. 6.3a,d). The later method is cheap, easy to implement and very popular amongst researchers attempting to dehydrate whilst collecting X-ray data in-house and for simple cryo-cooling (Yao et al. 2004; Kim et al. 2007). As the mounts (MicroRT system - MiTeGen) are standard, the technique is also available at any synchrotron beamline where manual mounting is possible. It is most frequently used as a quick dehydration method and as an easy way of keeping samples stable during room temperature data collections.

The process involves pre-loading (using a gelloading tip) a transparent polymer capillary that is sealed at one end with a small volume of stabilising or dehydrating solution. Crystals are harvested with a loop or mesh mounted onto a pin and then covered with the capillary through the open end such that it seals. The sample is maintained at a close distance from the liquid. The pin can then be mounted and data collected through the capillary whilst the crystal is kept stable or whilst being dehydrated. The dehydration rate is dependent on the solution composition, the sample volume, the solution volume and the distance between the sample and solution. Cryo-cooling is possible at any point by quickly removing the capillary and plunging in liquid nitrogen (Kalinin and Thorne 2005; Warkentin et al. 2008).

\subsubsection{Dehydration Using the FMS}

The Free Mounting System (FMS) was the first device able to automate the process of dehydration. It operates based on the alteration of $\mathrm{RH}$ by the adjustment of the dew point and is able to regulate humidity in a crystal from $98 \%$ to $50 \%$ at temperatures ranging from $15{ }^{\circ} \mathrm{C}$ to $25{ }^{\circ} \mathrm{C}$. It has been used in some academic laboratories and private companies yielding interesting results (Kiefersauer et al. 2000a, b).

The key feature of the device is a holding block (Fig. 6.3b,e) that is able to keep the temperature of the sample and the surrounding airflow stable. The samples are mounted onto meshes or loops and placed inside the block. The block is then placed over a standard goniometer mount to allow users to centre the samples onto the Xray beam path and collect data whilst dehydrating samples.

The major advantage of this device over classic methods is that complex protocols such 
a
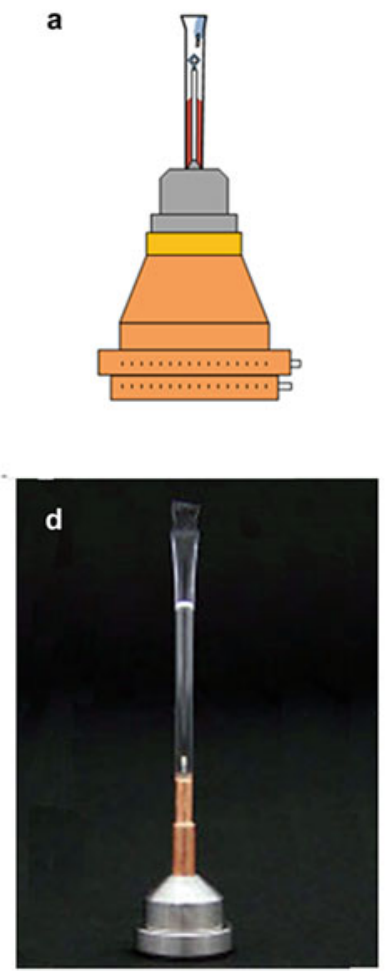

b
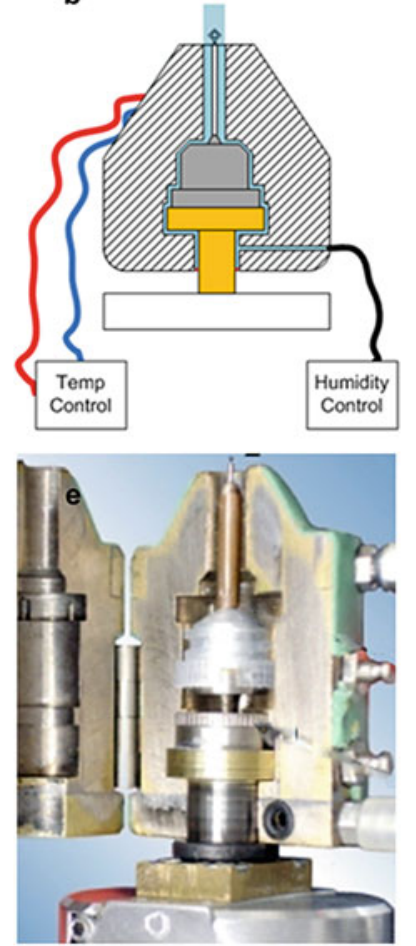
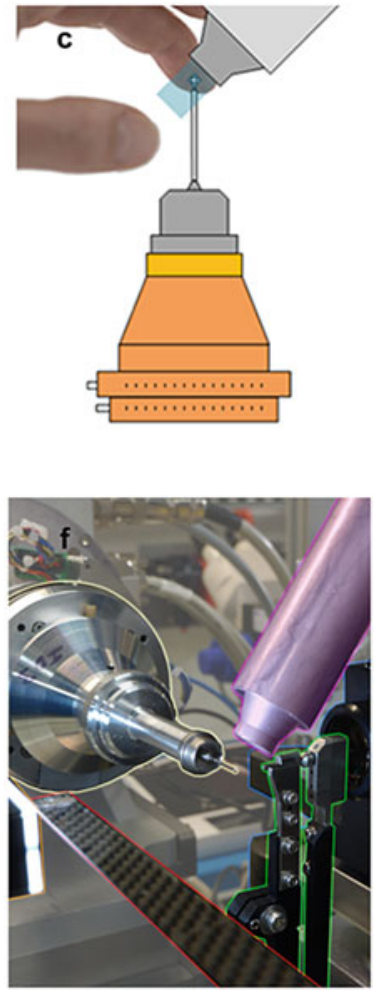

Fig. 6.3 Methods of dehydration with X-ray feedback. (a) and (d) Capillary dehydration. (b) and (e) Free Mounting System. (c) and (f) The humidity control dehydration device (HC1)

gradient dehydration, rehydration and reverse hydration can be achieved with a high level of reproducibility while at the same time allowing diffraction feedback. The only disadvantage compared to the classic method is the fact that only one or two crystals can be analysed at the same time.

The FMS has been used in a number of successful cases, most notably the improvement in diffraction of the F1-ATPase protein (Bowler et al. 2006). It also has allowed for the determination of the extremely interesting case of carbon monoxide oxidase (CODH) (Kiefersauer et al. 2000a, b) that required a complex dehydrationrehydration process only possible with the use of the FMS.

Despite its success, the complexity of sample handling and bulky design have prevented FMS from being widely used in laboratories worldwide and also making synchrotrons sources shy away from installing it on macromolecular crystallog- raphy (MX) beamlines. Lately the developers of the device have also proved it can be used in combination with a heating laser. This gives rise to new alternate options to automate dehydration and new cryo-cooling methods, but no new crystal structures have as yet resulted from this sort of work (Kiefersauer et al. 2014).

\subsubsection{Dehydration Using the HC1 System}

The humidity control dehydration device (HC1) was developed a few years after the FMS in order to address the factors limiting the wider use of this method by the community (SanchezWeatherby et al. 2009; Russi et al. 2011). The $\mathrm{HC} 1$ key difference is that, despite also being based on dew point, the delivery of humid airflow is achieved via a nozzle rather than a cooling block (Fig. 6.3c,f). The HC1 nozzle makes it unable to alter the temperature of the air around the sample but makes it very easy to install and 
use it in a standard academic laboratory with a home X-ray source or any MX synchrotron beamline. The low complexity of the device reduced the cost and simplified its maintenance making it better received by many academic laboratories and a large number of synchrotron beamlines around the world. To date nine devices are available at several world synchrotrons. The ESRF/EMBL/ILL operate two devices available across the MX beamlines. The HelmholtzZentrum Berlin (Mueller et al. 2012) and Max IV Laboratory (Ursby et al. 2013) operate the device installed in one of their MX branches. In the US, both APS (NE-CAT 24-ID-E) and the ALS (Berkeley Center for Structural Biology beamlines) have one each, the Canadian Light Source (Fodje et al. 2014) has one and Diamond Light Source in the UK has another two that can be both installed in any of the MX beamlines and also in a laboratory as an off-line setup.

Considerable efforts have been made at several of the synchrotrons in order to incorporate the $\mathrm{HC} 1$ within their hardware and software. For example, workflows within MxCuBE (Gabadinho et al. 2010) have been adapted to undertake dehydration experiments (Brockhauser et al. 2012) and the Diamond beamline software (GDA) now includes a bespoke data collection perspective solely dedicated at undertaking these sorts of experiments. This allows synchrotron users to be able to undertake a range of dehydration experiments as part of their normal beamtime allocation. In some synchrotron facilities (BM14 at the ESRF and I02, I03 and I04 at Diamond), installations also include rapid swapping between the use of the $\mathrm{HC} 1$ and the standard cryo-temperature allowing constant routine use without the local beamline support. Others (like MaxLab and BESSY) have opted for a more permanent installation (Mueller et al. 2012; Ursby et al. 2013).

Either in the laboratory or at the synchrotron beamline, any $\mathrm{HC} 1$ experiment requires the mounting of samples in the airflow at their starting Relative Humidity (RHi). The relative humidity is empirically determined by observing the behaviour of a droplet solution (crystallisation solution) under the humid airstream while varying the $\mathrm{RH}$. Once the optimum humidity has been determined, crystal samples can be mounted using micromeshes (MiTeGen) so that any excess buffer can be removed by wicking. This makes the experiments more reproducible and ensures samples don't move during the process.

Dehydration protocols can then be applied to each sample by starting at the RHi and lowering humidity to desired target points. At these points samples can either be directly analysed with Xrays or cryo-cooled for later analysis. The key to any experiment is to try to induce changes in the crystal lattice that will yield a better diffracting sample. Once a potential dehydration protocol has been determined then a number of crystals can be dehydrated and cryo-protected to find the elusive structure. If available, this final harvest can be undertaken in a laboratory in order to save precious beam time and improve throughput.

When using the $\mathrm{HCl}$ in a beamline, as it is a room temperature experiment, it is important to try to separate the effects from dehydration from the expected effects of radiation damage. A burn test may be appropriate to define a baseline. The best way of following an on-line dehydration experiment is to collect weak, wide oscillation images $\left(1-2^{\circ}\right)$ that will allow manual indexing to determine lattice dimensions and mosaicity. Following the changes in the lattice, the researcher can determine any transition points of interest that could suggest points of improved diffraction.

If undertaking the experiment in the laboratory it is important to also harvest some control samples under known conditions and try to ensure samples have had enough time to undergo any transition (10-15 min at least) prior to cooling. Harvesting a number of repeats helps to assess the true final outcomes of the process giving an indication of the likeliness of the method in study.

\subsubsection{In situ Dehydration}

In most of the MX beamlines across the world, sample environments (also called experimental hutches) have been made more automationfriendly. Consequently, the space available for additional and special setups became more restrictive. Despite this, due to the developments of photon counting pixel array detectors and their potential to help outrun radiation damage 
Fig. 6.4 Diagram showing the different stages required for an in situ dehydration experiment
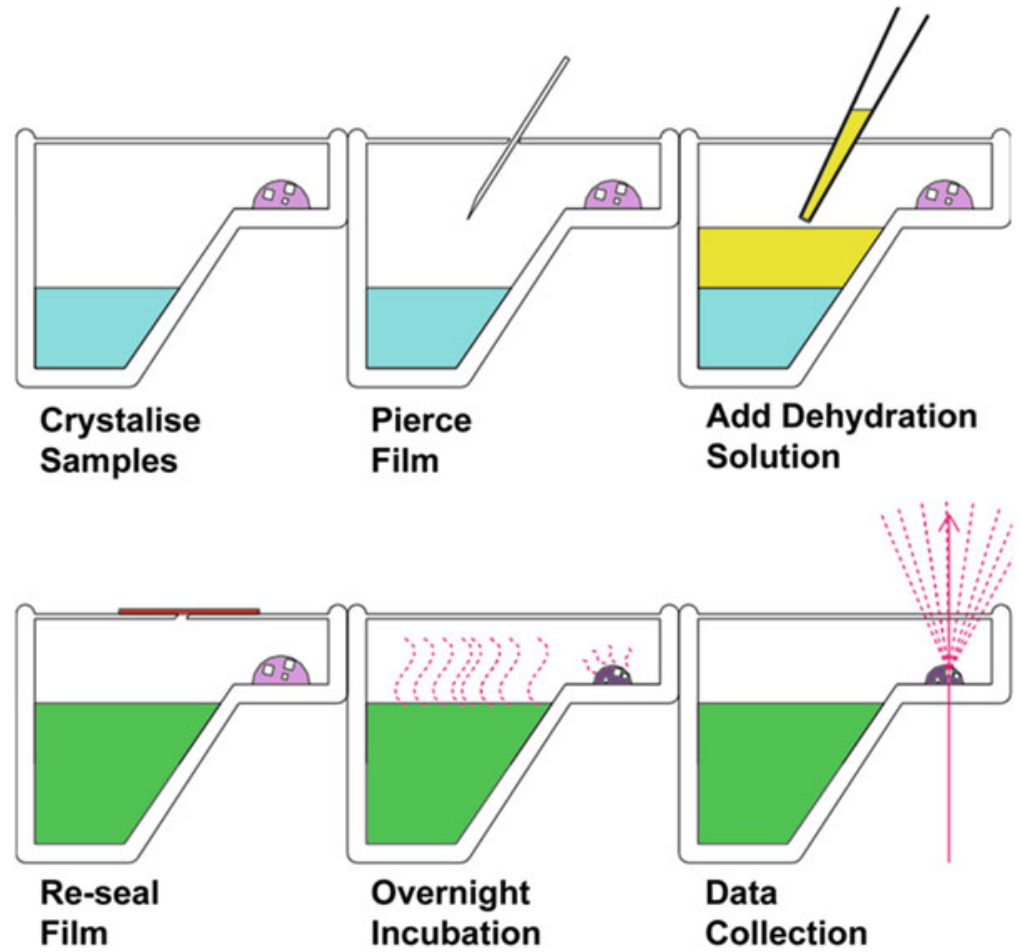

(Kraft et al. 2009; Aishima et al. 2010; Rajendran et al. 2011) there have been a number of technical upgrades to allow data collection of room temperature samples within these crowded spaces. These include new crystallisation plates (with lower profile and better geometry), upgraded goniometry, dedicated robotics and imaging systems, make room temperature data collection possible. The option of collecting data directly from crystallisation plates without the need of any physical manipulation of the crystals brings new links between the experiment in the laboratory and the X-ray source. Essentially, in this method, crystals from the same crystallisation condition are grown in a full 96-well crystallisation plate (always advisable to use crystallisation plates with flat bottoms to avoid visual artefacts when mounted at the beamline). Once crystals are grown, gradients of different dehydration solutions are added to the plate wells through small pierces (without touching the crystal drops) and re-sealed (see Fig. 6.4). The dehydration is allowed to proceed for a period of $8-12 \mathrm{~h}$ before the plate be taken to an X-ray source with capabilities for in situ plate screening (Douangamath et al. 2013; Moraes and Archer 2015; Axford et al. 2015). This method is essentially a vapour control, by exchanging well solutions, which allows multiple samples to be dehydrated in a number of ways in one single procedure. With high throughput tools it is possible, with a reasonable amount of beam time, to analyse these complex dehydration results without the blurring effect of cryo-cooling. In summary, the fundamental advantages of using in situ is that there is no need for direct crystal handling and therefore small or delicate samples are not a problem, crystals can grow at $4{ }^{\circ} \mathrm{C}$ with the procedure carrying on at the same temperature and there is no need for cryo-cooling.

In these sorts of experiments, the key is to analyse large populations of crystals and observe general trends of change amongst them. These experiments are analysed by studying changes in lattice dimensions, mosaicity, resolution and diffraction properties as a function of the chemical composition of wells. The nature of the initial cohort of crystals can be well defined; therefore their susceptibility to change, any transition 
points, any regions of stability and a minimum lattice size should all be possible using limited beamtime. Once points of interest are identified, a sub-population of test samples can then be harvested and cryo-cooled in order to confirm any beneficial effects. These cryo-cooling tests should help define a best protocol for dehydration and cryo-protection.

\subsubsection{Analysing Results and Making Decisions}

As it has been described throughout this chapter, there are a number of valid options when carrying out dehydration. The researcher's choice will depend on a number of constraints such sample, resources and time availability (see Table 6.4). Also depending on the method used the outcomes will vary. In certain occasions, observations will be immeasurable due to crystal cracking, shrinking or dissolving, in others observations will be easy to measure (lattice dimensions, mosaicity and resolution limits) and unfortunately, in others negative results will be the outcome (lack of diffraction, presence of ice rings or non indexable lattices). For these reasons it is advisable to correlate all the different information available and try to make sense of partial data. All the different types of dehydration experiments can be linked via their relation to Relative Humidity. By using available tabulated information (salt dehydration) or measuring empirical humidity (HC1/FMS) researchers can correlate results from different experiments. The idea is to try to extract as much information and try new experiments that may lead to a positive outcome with the available tools. For example, prior to any dehydration attempt, most researchers have already tried standard cryo-cooling, soaks and handled their crystals in a number of ways. Researchers are familiar with crystals stability under the microscope and their methods for soaking in cryo-protectant are useful when planning dehydration protocols either using the HC1/FMS or a vapour diffusion experiment. Similarly, measuring or calculating the equilibrium RH of these solutions can give to the crystallographer clues of why some of the solutions are better than others and suggest new cooling protocols. Often, cryo diffraction data is also available and so a quick comparison with room temperature data collection can possibly yield fruitful clues. Variable indexing in cryo lattices can be an indication of a varied initial crystal population suggesting a variable response to cryo making crystals interesting candidates for dehydration. A review of old crystal forms observed during past experiments (despite not diffracting well) can also give useful clues to plan dehydration experiments. During dehydration, the observation of lattice transitions, minimum lattices and points of loss of diffraction can be used later when defining starting points for cryo-cooling methods. Even dehydration itself can be used as a simple cryo-protecting strategy useful in preventing adverse effects of penetrative cryo-protectant (Pellegrini et al. 2011). Using Relative Humidity as a way of cross-referencing results from the different experiments allows decisions to be made on quantitative measurements and permits experiment planning that will yield easier to compare results.

As it was mentioned earlier, when attempting dehydrating membrane protein crystals it is worth keeping in mind the particular packing of these crystals and how this might affect the results. Detergent micelle size/concentration and limited hydrophilic contacts between the molecules in the crystal lattice should be the main points to take into account during interpretation of results. In addition, as these crystals have larger solvent channels, often require greater dehydration than required for soluble proteins. Therefore, one should include both vapour diffusion and chemical modification methods as they might yield markedly different results. Finally, given the fragile nature these crystals, they are likely to benefit from handling free techniques such as in situ dehydration and dialysis.

\subsection{Conclusions: Success in Dehydration}

Unfortunately due to a multitude of constraints in most research projects, controlled dehydration is often not attempted or just coarsely tested. Researchers frequently associate the positive 


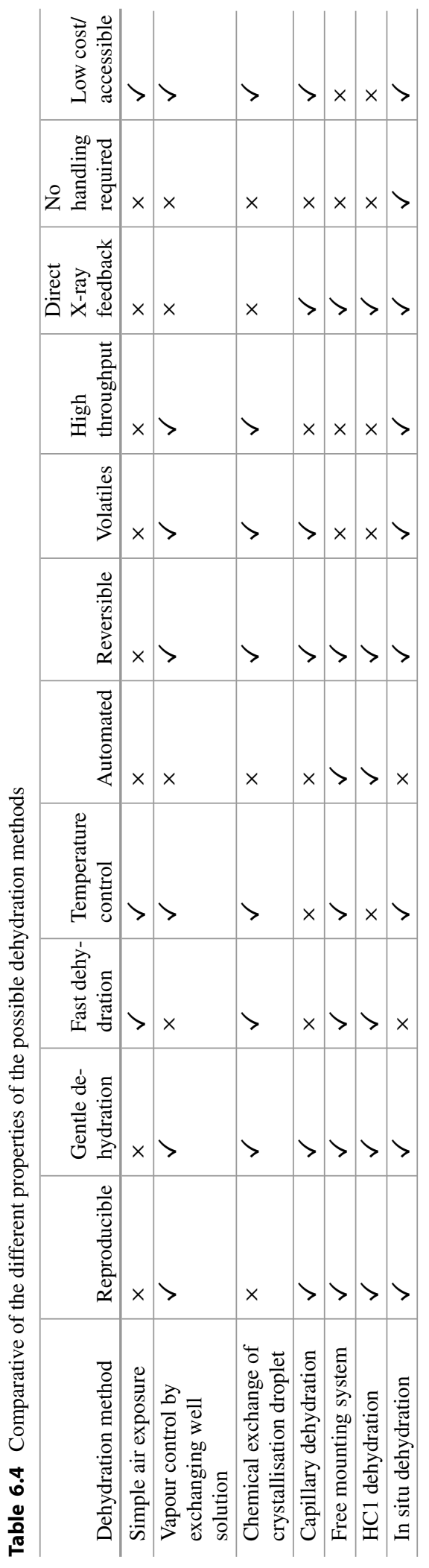


outcomes of dehydration with the time required for the experiment. Later, by asking colleagues and consulting the available literature often conclude that it is unlikely to be useful for them. This makes dehydration very much a last resort technique, in particular when more standard approaches have failed and the project is important enough to pursue alternatives. In addition, it is known that many of dehydration success cases are not reported in methods sections of scientific papers and it is considered of less importance.

With regard to membrane proteins, a recent review (Bowler et al. 2015; Russo et al. 2012) on dehydration showed that despite the large number of soluble protein structures solved compared with membrane proteins, the number of reported membrane protein targets facilitated by dehydration seem to be very similar to soluble proteins, suggesting that success is inclined towards membrane proteins. It is difficult to be sure but three potential explanations for this phenomenon are:

- Membrane proteins are more costly (in time and money) to produce and investigators may be willing to try every available technique possible before moving onto other projects

- The nature of the membrane protein crystals themselves could be the blame. Membrane protein crystals have smaller water content but overall higher solvent content facilitating motions across the solvent channels making them more susceptible to improvement by dehydration

- It seems that a membrane protein structure which is facilitated by a dehydration method always has a reference within the results section of the scientific paper as it is perceived to be important for the research field

Whatever the reason, it is clear that undertaking controlled dehydration in membrane protein crystals is well worth the effort in most cases.

Acknowledgments We gratefully acknowledge the Diamond Light Source Ltd and the Membrane Protein Laboratory (Wellcome Trust (grant 099165/Z/12/Z).

\section{References}

Abergel C (2004) Spectacular improvement of X-ray diffraction through fast desiccation of protein crystals. Acta Crystallogr D Biol Crystallogr 60(8):1413-1416

Adachi H, Umena Y, Enami I, Henmi T, Kamiya N, Shen JR (2009) Towards structural elucidation of eukaryotic photosystem II: purification, crystallization and preliminary X-ray diffraction analysis of photosystem II from a red alga. Biochim Biophys Acta 1787(2): 121-128

Aishima J, Owen RL, Axford D, Shepherd E, Winter G et al (2010) High-speed crystal detection and characterization using a fast-readout detector. Acta Crystallogr D Biol Crystallogr 66(9):1032-1035

Alcorn T, Juers DH (2010) Progress in rational methods of cryoprotection in macromolecular crystallography. Acta Crystallogr D Biol Crystallogr 66:366-373

Amunts A, Drory O, Nelson N (2007) The structure of a plant photosystem I supercomplex at 3.4 A resolution. Nature 447(7140):58-63

Axford D, Foadi J, Hu NJ, Choudhury HG, Iwata S et al (2015) Structure determination of an integral membrane protein at room temperature from crystals in situ. Acta Crystallogr D Biol Crystallogr 71(6):0

Basavappa R, Petri ET, Tolbert BS (2003) A quick and gentle method for mounting crystals in capillaries. $\mathbf{J}$ Appl Crystallogr 36(5):1297-1298

Biswal BK, Vijayan M (2002) Structures of human oxyand deoxyhaemoglobin at different levels of humidity: variability in the $\mathrm{T}$ state. Acta Crystallogr D Biol Crystallogr 58(7):1155-1161

Bowler MW, Montgomery MG, Leslie AG, Walker JE (2006) Reproducible improvements in order and diffraction limit of crystals of bovine mitochondrial F(1)-ATPase by controlled dehydration. Acta Crystallogr D Biol Crystallogr 62(9):991-995

Bowler MW, Mueller U, Weiss MS, Sanchez-Weatherby J, Sorensen TLM et al (2015) Automation and experience of controlled crystal dehydration: results from the European synchrotron HC1 collaboration. Cryst Growth Des 15(3):1043-1054

Brockhauser S, Svensson O, Bowler MW, Nanao M, Gordon E, Leal RM, Popov A, Gerring M, McCarthy AA, Gotz A (2012) The use of workflows in the design and implementation of complex experiments in macromolecular crystallography. Acta Crystallogr D Biol Crystallogr 68(8):975-984

Caffrey M (2003) Membrane protein crystallization. J Struct Biol 142(1):108-132

Dobrianov I, Kriminski S, Caylor CL, Lemay SG, Kimmer C, Kisselev A, Finkelstein KD, Thorne RE (2001) Dynamic response of tetragonal lysozyme crystals to changes in relative humidity: implications for postgrowth crystal treatments. Acta Crystallogr D Biol Crystallogr 57(1):61-68

Douangamath A, Aller P, Lukacik P, Sanchez-Weatherby J et al (2013) Using high-throughput in situ plate screening to evaluate the effect of dehydration on protein crystals. Acta Crystallogr D Biol Crystallogr 69(5):920-923 
Esnouf RM, Ren J, Garman EF, Somers DO, Ross CK, Jones EY, Stammers DK, Stuart DI (1998) Continuous and discontinuous changes in the unit cell of HIV-1 reverse transcriptase crystals on dehydration. Acta Crystallogr D Biol Crystallogr 54(5): 938-953

Farley C, Burks G, Siegert T, Juers DH (2014) Improved reproducibility of unit-cell parameters in macromolecular cryocrystallography by limiting dehydration during crystal mounting. Acta Crystallogr D Biol Crystallogr 70(8):2111-2124

Fodje M, Grochulski P, Janzen K, Labiuk S, Gorin J et al (2014) 08B1-1: an automated beamline for macromolecular crystallography experiments at the Canadian Light Source. J Synchrotron Rad 21(3):633-663

Gabadinho J, Beteva A, Guijarro M, Rey-Bakaikoa V, Spruce D, Bowler MW, Brockhauser S, Flot D, Gordon EJ, Hall DR, Lavault B, McCarthy AA, McCarthy J, Mitchell E, Monaco S, Mueller-Dieckmann C, Nurizzo D, Ravelli RB, Thibault X, Walsh MA, Leonard GA, McSweeney SM (2010) MxCuBE: a synchrotron beamline control environment customized for macromolecular crystallography experiments. J Synchrotron Radiat 17(5):700-707

Garman E (1999) Cool data: quantity AND quality. Acta Crystallogr D Biol Crystallogr 55(10):1641-1653

Greenspan L (1977) Humidity fixed points of binary saturated aqueous solutions. J Res Natl Inst Stand Technol 81A(1):89-96

Hellert J, Krausze J, Schulz TF, Luhrs T (2014) Crystallization, room-temperature $\mathrm{X}$-ray diffraction and preliminary analysis of Kaposi's sarcoma herpesvirus LANA bound to DNA. Acta Crystallogr F 70(11):1570-1574

Heras B, Martin JL (2005) Post-crystallization treatments for improving diffraction quality of protein crystals. Acta Crystallogr D Biol Crystallogr 61(9):1173-1180

Hope H (1988) Cryocrystallography of biological macromolecules: a generally applicable method. Acta Crystallogr B 44(1):22-26

Hu NJ, Iwata S, Cameron AD, Drew D (2011) Crystal structure of a bacterial homologue of the bile acid sodium symporter ASBT. Nature 478(7369):408-411

Huxley HE, Kendrew JC (1953) Discontinuous lattice changes in haemoglobin crystals. Acta Crystallogr 6(1):76-80

Juers DH, Matthews BW (2004) Cryo-cooling in macromolecular crystallography: advantages, disadvantages and optimization. Q Rev Biophys 37(2):105-119

Kalinin Y, Thorne R (2005) Crystal growth in X-raytransparent plastic tubing: an alternative for highthroughput applications. Acta Crystallogr D Biol Crystallogr 61(11):1528-1532

Kalinin Y, Kmetko J, Bartnik A, Stewart A, Gillilan R, Lobkovsky E, Thorne R (2005) A new sample mounting technique for room-temperature macromolecular crystallography. J Appl Crystallogr 38(2):333-339

Kaushal PS, Sankaranarayanan R, Vijayan M (2008) Water-mediated variability in the structure of relaxed- state haemoglobin. Acta Crystallogr Sect F Struct Biol Cryst Commun 64(6):463-469

Kiefersauer R, Than ME, Dobbek H, Gremer L, Melero $M$ et al (2000a) A novel free-mounting system for protein crystals: transformation and improvement of diffraction power by accurately controlled humidity changes. J Appl Crystallogr 33(5):1223-1230

Kiefersauer R, Dobbek H, Grazulis S, Than M, Huber $\mathrm{R}$ (2000b) A novel free-mounting system for protein crystals: transformation and improvement of diffraction power by accurately controlled humidity changes. Acta Crystallogr Sect A 56(s1):s441

Kiefersauer R, Dobbek H, Than ME, Huber R (2002) A novel free-mounting system for protein crystals: improvement of diffraction power by accurately controlled humidity changes. Acta Crystallogr Sect A 58(s1):c 150

Kiefersauer R, Grandl B, Krapp S, Huber R (2014) IR laser-induced protein crystal transformation. Acta Crystallogr D Biol Crystallogr 70(5): 1224-1232

Kim CU, Hao Q, Gruner SM (2007) High-pressure cryocooling for capillary sample cryoprotection and diffraction phasing at long wavelengths. Acta Crystallogr D Biol Crystallogr 63(5):653-659

Kraft P, Bergamaschi A, Broennimann C, Dinapoli R, Eikenberry EF et al (2009) Performance of singlephoton-counting PILATUS detector modules. J Synchrotron Rad 16(3):368-375

Kuo A, Bowler MW, Zimmer J, Antcliff JF, Doyle DA (2003) Increasing the diffraction limit and internal order of a membrane protein crystal by dehydration. J Struct Biol 141(2):97-102

Mac Sweeney A, D'Arcy A (2003) A simple and rapid method for mounting protein crystals at room temperature. J Appl Crystallogr 36(1):165-166

Mitchell EP, Garman EF (1994) Flash freezing of protein crystals: investigation of mosaic spread and diffraction limit with variation of cryoprotectant concentration. J Appl Crystallogr 27(6):1070-1074

Moraes I, Archer M (2015) Methods for the successful crystallization of membrane proteins. In: Structural proteomics. Springer, New York, pp 211-230

Mueller U, Darowski N, Fuchs MR, Forster R, Hellmig M, Paithankar KS, Puhringer S, Steffien M, Zocher G, Weiss MS (2012) Facilities for macromolecular crystallography at the Helmholtz-Zentrum Berlin. J Synchrotron Radiat 19(3):442-449

Nagendra HG, Sukumar N, Vijayan M (1998) Role of water in plasticity, stability, and action of proteins: the crystal structures of lysozyme at very low levels of hydration. Proteins 32(2):229-240

Newman J (2006) A review of techniques for maximizing diffraction from a protein crystal in stilla. Acta Crystallogr D Biol Crystallogr 62(1):27-31

Pellegrini E, Piano D, Bowler MW (2011) Direct cryocooling of naked crystals: are cryoprotection agents always necessary? Acta Crystallogr D Biol Crystallogr 67(10):902-906 
Perutz MF (1946) The composition and swelling properties of haemoglobin crystals. Trans Faraday Soc 42:B187-B195

Pflugrath J (2015) Practical macromolecular cryocrystallography. Acta Crystallogr Sect F Struct Biol Cryst Commun 71(6):622-642

Pickford MG, Garman EF, Jones EY, Stuart DI (1993) A design of crystal mounting cell that allows the controlled variation of humidity at the protein crystal during X-ray diffraction. J Appl Crystallogr 26(3):465-466

Rajendran C, Dworkowski FS, Wang M, Schulze-Briese C (2011) Radiation damage in room-temperature data acquisition with the PILATUS 6M pixel detector. J Synchrotron Radiat 18(3):318-328

Rockland LB (1960) Saturated salt solutions for static control of relative humidity between 5 and $40{ }^{\circ} \mathrm{C}$. Anal Chem 32(10):1375-1376

Russi S, Juers DH, Sanchez-Weatherby J, Pellegrini E, Mossou E et al (2011) Inducing phase changes in crystals of macromolecules: status and perspectives for controlled crystal dehydration. J Struct Biol 175(2):236-243

Russo KI, Sica F, Mattia CA, Merlino A (2012) Increasing the X-ray diffraction power of protein crystals by dehydration: the case of bovine serum albumin and a survey of literature data. Int J Mol Sci 13(3):3782-3800

Sanchez-Weatherby J, Bowler MW, Huet J, Gobbo A, Felisaz F et al (2009) Improving diffraction by humidity control: a novel device compatible with X-ray beamlines. Acta Crystallogr D Biol Crystallogr 65(12):1237-1246

Saraswathi NT, Sankaranarayanan R, Vijayan M (2002) Effect of stabilizing additives on the structure and hydration of proteins: a study involving monoclinic lysozyme. Acta Crystallogr D Biol Crystallogr 58(7):1162-1167
Shi J, Sivaraman J, Song J (2008) Mechanism for controlling the dimer-monomer switch and coupling dimerization to catalysis of the severe acute respiratory syndrome coronavirus 3C-like protease. J Virol 82(9):4620-4629

Sukumar N, Biswal BK, Vijayan M (1999) Structures of orthorhombic lysozyme grown at basic $\mathrm{pH}$ and its low-humidity variant. Acta Crystallogr D Biol Crystallogr 55(4):934-937

Ursby T, Unge J, Appio R, Logan DT, Fredslund F, Svensson C, Larsson K, Labrador A, Thunnissen MM (2013) The macromolecular crystallography beamline I911-3 at the MAX IV laboratory. J Synchrotron Radiat 20(4):648-653

Warkentin M, Stanislavskaia V, Hammes K, Thorne RE (2008) Cryocrystallography in capillaries: critical glycerol concentrations and cooling rates. J Appl Crystallogr 41(4):791-797

Wheeler MJ, Russi S, Bowler MG, Bowler MW (2012) Measurement of the equilibrium relative humidity for common precipitant concentrations: facilitating controlled dehydration experiments. Acta Crystallogr Sect F Struct Biol Cryst Commun 68(1): 111-114

Winston PW, Bates DH (1960) Saturated solutions for the control of humidity in biological research. Ecology 1:232-237

Yadav MK, Gerdts CJ, Sanishvili R, Smith WW, Roach LS, Ismagilov RF, Kuhn P, Stevens RC (2005) In situ data collection and structure refinement from microcapillary protein crystallization. J Appl Crystallogr 38(6):900-905

Yao M, Yasutake Y, Tanaka I (2004) Flash-cooling of macromolecular crystals in a capillary to overcome increased mosaicity. Acta Crystallogr D Biol Crystallogr 60(1):39-45

Open Access This chapter is licensed under the terms of the Creative Commons Attribution 4.0 International License (http://creativecommons.org/licenses/by/4.0/), which permits use, sharing, adaptation, distribution and reproduction in any medium or format, as long as you give appropriate credit to the original author(s) and the source, provide a link to the Creative Commons license and indicate if changes were made.

The images or other third party material in this chapter are included in the chapter's Creative Commons license, unless indicated otherwise in a credit line to the material. If material is not included in the chapter's Creative Commons license and your intended use is not permitted by statutory regulation or exceeds the permitted use, you will need to obtain permission directly from the copyright holder. 